\title{
Non-steroidal anti-inflammatory drugs prescribing in chronic kidney disease: an observational study
}

\author{
Sunil Bhopal ${ }^{1}$, James Chan ${ }^{1}$, Oliver Ellis ${ }^{1}$, Sarah Graham ${ }^{1}$, Stephen Halpin ${ }^{1}$, Thomas Lawrence ${ }^{1}$, \\ Joseph Laws ${ }^{1}$ and Anne-Marie Howes ${ }^{2}$ \\ ${ }^{1}$ School of Medicine, University of Leeds, Leeds, UK \\ ${ }^{2}$ Academic Unit of Primary Care, Leeds Institute of Health Sciences, Leeds, UK
}

\begin{abstract}
Aim: This study assessed the prescription of potentially nephrotoxic non-steroidal antiinflammatory drugs (NSAIDs) to patients with chronic kidney disease (CKD) in general practice. Background: CKD poses a considerable disease burden in the UK. Guidelines state that caution should be exercised when prescribing NSAIDs to CKD patients, due to increased risk of rapid kidney disease progression. Methods: We reviewed the medical records of 1427 patients with CKD Stages 3-5 in seven general practices in West Yorkshire. Findings: A total of $792(55.5 \%)$ were prescribed NSAIDs; $128(9 \%)$ of these were prescribed NSAIDs excluding low-dose aspirin. Twenty-three (20.2\%) patients who were prescribed NSAIDs had no record of CKD monitoring in the preceding year. Conclusion: Prescription of NSAIDs is likely to be contributing to unnecessary renal impairment.
\end{abstract}

Key words: family practice; chronic kidney disease; NSAIDs; prescribing

Received 14 June 2009; accepted 25 November 2009; first published online 10 March 2010

\section{How this fits in}

A total of $8.5 \%$ of the UK population is affected by chronic kidney disease (CKD), and worsening of the condition imposes a high burden of morbidity and mortality. Non-steroidal anti-inflammatory drugs (NSAIDs) are commonly prescribed medications and their use is associated with increased risk of rapid kidney disease progression. This study found that NSAIDs were often prescribed and many patients were poorly monitored against National Institute for Clinical Excellence (NICE) guidelines. Prescribers should exercise greater caution when prescribing NSAIDs to patients with CKD.

\section{Introduction}

Chronic kidney disease (CKD) is diagnosed by two measurements of estimated glomerular filtration rate

Correspondence to: Thomas Lawrence, Room 7.09 Worsley Building, University of Leeds, Leeds, LS2 9JT, UK. Email: Tom.Lawrence@doctors.org.uk

(C) Cambridge University Press 2010
(eGFR units: $\mathrm{mL} / \mathrm{min} / 1.73 \mathrm{~m}^{2}$ ) at least 3 months apart. CKD stages 1 and 2 require signs of kidney damage on other tests, whilst CKD stages 3-5 are defined on the basis of eGFR alone (CKD stage 3, eGFR 30-59; CKD stage 4, eGFR 15-29; CKD stage 5, eGFR less than 15). CKD stages 1-5 (CKD1-5) represent increasing degrees of renal impairment. In UK, $8.5 \%$ of the population is affected by CKD (Stevens et al., 2007). Progression from CKD1-4 to CKD5 (renal failure) imposes a high burden of morbidity and mortality: over $2 \%$ of the total National Health Service budget is spent on renal replacement therapy (NICE, 2008); renal transplants cost around $£ 20000$ per patient, with an ongoing cost of $£ 6500$ per patient per year (Davies and Rodderick, 1997). Only a minority of patients will develop end-stage renal disease, but minor renal impairment is an independent risk factor for all causes of cardiovascular disease and mortality (Gomez et al., 2006). Effective management of CKD in primary care is vital in preventing progression to CKD5 (renal failure), and controlling the disease burden posed by cardiovascular and other diseases. 
Non-steroidal anti-inflammatory drugs (NSAIDs) are amongst the most commonly prescribed drugs in the world. NSAID use has been associated with acute interstitial nephritis and worsening of CKD (Fored et al., 2001; House et al., 2007). High cumulative NSAID exposure is associated with increased risk of rapid CKD progression (Gooch et al., 2007). However, not all NSAIDs present an equal risk. NICE guidance states that antiplatelet drugs should still be offered to people with CKD for the secondary prevention of Cardiovascular Disease, and that CKD is not a contraindication to the use of lowdose aspirin. Furthermore, guidance from the Renal Association suggests that low-dose aspirin may actually be underutilised in CKD, and that it does not cause progression of the disease (The Joint Specialty Committee on Renal Medicine of the Royal College of Physicians and the Renal Association, 2006).

NSAIDs were categorized as 'standard dose NSAIDs' or 'low-dose aspirin'. Low-dose aspirin was considered to be a dose of up to $100 \mathrm{mg} / \mathrm{day}$, as this is the dose used in the First United Kingdom Heart and Renal Protection study, which informed the Renal Association guidance (Baigent et al., 2005). 'Standard dose NSAIDs' refers to all NSAIDs other than low-dose aspirin.

NICE guidelines published in September 2008 recommend 'caution' when prescribing NSAIDs for patients with CKD over a prolonged period of time and recommend that eGFR should be monitored, particularly in those patients with greater risk of disease progression. Elsewhere in the guidance, it is suggested that eGFR should be checked regularly depending on disease stage (CKD1-2, every 12 months; CKD3, every 6 months; CKD4, every 3 months) (NICE, 2008). Our aim was to assess the quality of General Practitioner prescribing in CKD3-5, focusing on NSAID prescribing and monitoring of these patients.

\section{Method}

Data were collected in an 8-week period between August and October 2008 at seven general practices across West Yorkshire, where the authors were based on their medical student clinical attachments. The data were extracted from electronic patient records. Patients diagnosed with CKD3-5, and those with prescriptions for NSAIDs, were identified by use of computerised Read Codes. A snapshot of all CKD patients and those currently prescribed NSAIDs at the time of data collection were selected in preference to historical data in order to identify current prescribing trends.

Basic demographic details were collected for each practice. In order to assess the extent of NSAID prescribing in CKD patients, data collected included diagnosis date and NSAID prescribed. To assess monitoring, the date and value of the most recent eGFR was recorded.

The study was reviewed and assessed by a senior board of academics and clinicians at the University of Leeds and deemed not to require ethical approval as it was judged to be an audit. Consent was sought, and given by the seven General Practices. Data was collected in accordance with Caldicott Principles (1997) and the Data Protection Act (1998). Practice and patient details were anonymised to maintain prescriber and patient confidentiality. Data were analysed in Microsoft Excel using descriptive statistics to calculate proportions of patients prescribed NSAIDs and time since last eGFR recorded.

\section{Results}

Results for six sites are presented for NSAID prescribing and eGFR monitoring. Site 4 was omitted from the analysis of NSAID prescribing (Figure 1) and site 3 was omitted from the analysis of eGFR monitoring (Figure 2) because of the unavailability of data.

\section{Patient identification}

A total of 1427 patients were identified as having CKD3-5 in six of the practices. The mean prevalence was $4.11 \%$. With an average age of 75.6 years, it can be expected that many CKD patients have significant co-morbidities. A large variation in practice size is noted (ranging from 3200 to 12600 patients), as well as a varied average age (ranging from 34 to 46 years) One site had a mean age 7 years lower than the average, being in an area heavily populated by students. At this site, $1.81 \%$ of patient had CKD, which was also lower than the average of $4.11 \%$.

\section{NSAID prescribing in CKD3-5}

A mean proportion of $55.5 \%$ of CKD3-5 patients across sites were prescribed NSAIDs (range 48.9-76.9\%). Eighty-one percent of these 


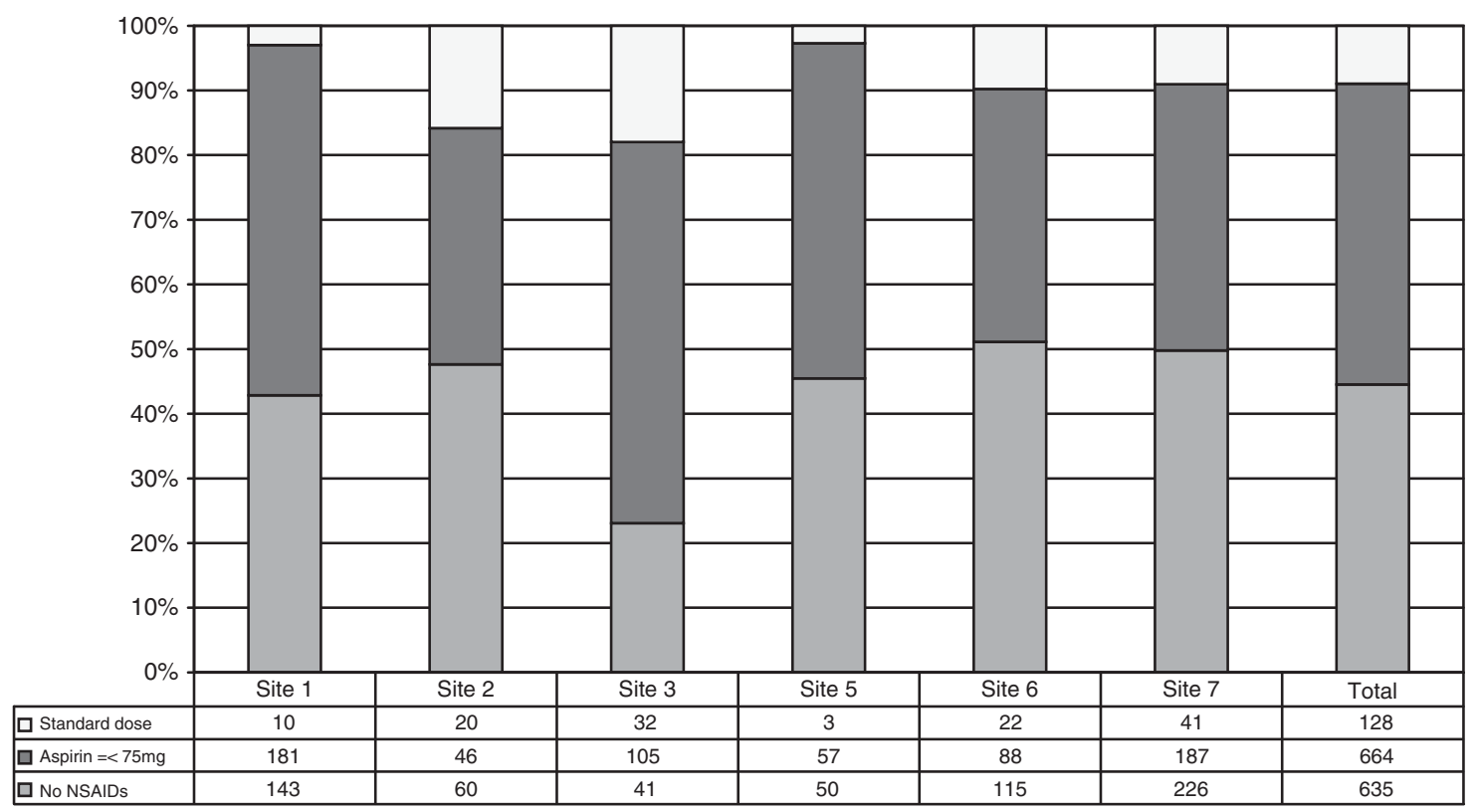

Figure 1 Prescription of low-dose aspirin and standard dose NSAIDs to CKD3-5 patients. NSAIDs, non-steroidal antiinflammatory drugs; CKD, chronic kidney disease

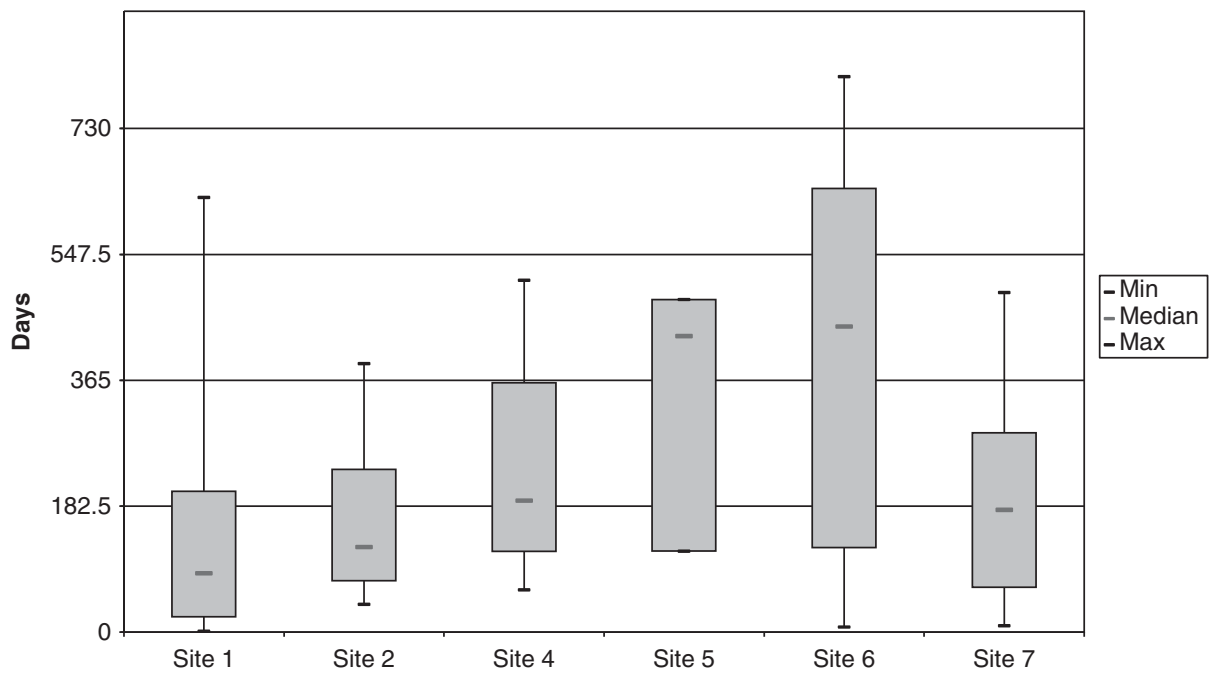

Figure 2 Box plot displaying days since last recorded eGFR for CKD3-5 patients being prescribed standard dose NSAIDs. eGFR, estimated glomerular filtration rate; CKD, chronic kidney disease; NSAIDs, non-steroidal antiinflammatory drugs

prescriptions were for aspirin $75 \mathrm{mg} /$ day; $47 \%$ of the total CKD3-5 patients $(n=664)$. The $19 \%$ of prescriptions for standard dose NSAIDs includes higher daily doses of aspirin. A mean of $9 \%$ (range 2.7-18\%) of CKD3-5 patients were prescribed standard dose NSAIDs (Figure 1). 
Table 1 Length of time (days) since last recorded estimated glomerular filtration rate in patients with Chronic kidney disease 3-5 on standard dose nonsteroidal anti-inflammatory drugs

\begin{tabular}{lcrll}
\hline Site & Number of patients & \multicolumn{3}{l}{ Last eGFR recorded } \\
\cline { 3 - 5 } & & $<1$ year & $1-2$ years & $2+$ years \\
\cline { 3 - 5 } & & $17(94.4)$ & $1(5.6)$ & $0(0)$ \\
1 & 18 & $19(95.0)$ & $1(5.0)$ & $0(0)$ \\
2 & 20 & $8(80.0)$ & $2(20.0)$ & $0(0)$ \\
4 & 10 & $1(33.3)$ & $2(66.7)$ & $0(0)$ \\
5 & 3 & $9(40.9)$ & $9(41.0)$ & $4(18.1)$ \\
6 & 22 & $37(90.2)$ & $4(9.8)$ & $0(0)$ \\
7 & 41 & $91(79.8)$ & $19(16.7)$ & $4(3.5)$ \\
Total & 114 & &
\end{tabular}

eGFR, estimated glomerular filtration rate.

\section{eGFR monitoring in CKD3-5 patients prescribed NSAIDs}

Sites with available data had some patients who had not been monitored for over a year (this data was not accessible from the computer database of one site). Across six sites, $20.2 \%(n=23)$ of CKD 3-5 patients prescribed standard-dose NSAIDs had not been monitored in the last year, with one practice having four patients who had not been monitored for over 2 years (Table 1). Additionally, two sites had median times since last eGFR readings of over 1 year (Figure 2).

\section{Discussion}

\section{Summary of main findings}

The mean CKD prevalence found is comparable with a reported national average of $5 \%$ (de Lusignan et al., 2005). A large proportion of these patients were found to be taking NSAIDs. The fact that across all sites, $9 \%$ of the CKD patients are on repeat prescription for standard dose NSAIDs indicates that some patients who are at risk of further kidney damage are regularly taking potentially nephrotoxic medications. Whilst there will be some valid indications for NSAID use in this often elderly population, proportions as high as $18 \%$ suggest that NSAID avoidance in these patients is often not prioritized.

The NICE guideline recommends that eGFR is measured at least annually in CKD patients being prescribed NSAIDs (NICE, 2008); however, this is not achieved in up to a fifth of CKD patients.
Fifteen patients did not have a recorded eGFR; although this could indicate an error with coding, it could also suggest a complete lack of monitoring for these patients.

Overall, it is likely that high levels of NSAID prescribing to patients with existing kidney disease is leading to avoidable nephrotoxicity, and that monitoring of this prescribing is often inadequate.

\section{Comparison with existing literature}

Rates of CKD within the study population fit with those found nationally. There is very little literature analysing prescribing practices. A 1998 analysis of prescribing in secondary care found that, contrary to British National Formulary guidelines, $10 \%$ of CKD patients were inappropriately prescribed NSAIDs (Wong and Jones, 1998).

\section{Strengths and limitations of the study}

Data were hand collected from both electronic and paper sources, using clearly pre-defined criteria. The overall prevalence of CKD3-5 was similar to the national prevalence found in the literature; this indicates that the study population was not atypical. Length of use of NSAIDs is visibly important; however, this study does not provide data on how long each prescription has existed, but differentiates long-term use from acute use of the medications only by collecting data exclusively from repeat prescriptions. This study does not investigate reasons behind prescribers' decisions, hence no conclusions can be drawn as to risk/benefit ratio for individual patients. The method relies on the use of pre-coded data, which may not be accurate. Sampling in this study was non-random. The unavailability of certain data from the various computerised data collection systems at different sites meant that all seven sites could not be included at all the stages of the analysis.

\section{Implications for clinical practice}

Low-dose aspirin is useful in prevention of cardiovascular disease in patients with CKD, but other NSAID use in patients with CKD results in faster progression of their disease. Thus, the health of some patients at study sites may be being adversely affected. Prescribers need to be more aware of implications in relation to CKD when deciding 
to prescribe NSAIDs, and more vigilant in their monitoring. CKD has received increased attention in the latest quality outcomes framework guidance, and consideration could be given to include eGFR monitoring as an indicator in future. If our findings were more widely transferable, it is probable that current clinical practice is exacerbating a considerable public health burden.

\section{Acknowledgements}

We would like to thank Dr Simon de Lusignan (St George's, University of London) and Professor Robbie Foy (Academic Unit of Primary Care, University of Leeds) for their comments on earlier drafts of this work, and Theresa Munyombwe (Biostatistics Unit, University of Leeds) for statistical advice. We would also like to thank the practice staff who assisted with early stages of data collection.

\section{References}

Baigent, C., Landray, M., Leaper, C., Altmann, P., Armitage, J., Baxter, A., Cairns, H., Collins, R., Foley, R. and Frighi, V. 2005: First United Kingdom Heart and Renal Protection (UK-HARP-I) study: biochemical efficacy and safety of simvastatin and safety of low-dose aspirin in chronic kidney disease. American Journal of Kidney Diseases 45, 473-84.

Davies, R. and Rodderick, P. 1997: Predicting the future demand for renal replacement therapy in England using simulation modelling. Nephrology Dialysis Transplantation 12, 2512-16.

de Lusignan, S., Chan, T., Stevens, P., O'Donoghue, D., Hague, N., Dzregah, B., Van Vlymen, J., Walker, M. and Hilton, S. 2005: Identifying patients with chronic kidney disease from general practice computer records. Family Practice 22, 234-41.

Fored, C.M., Ejerblad, E., Lindblad, P., Fryzek, J.P., Dickman, P.W., Signorello, L.B., Lipworth, L., Elinder, C.G., Blot, W.J., McLaughlin, J.K., Zack, M.M. and Nyren, O. 2001: Acetaminophen, aspirin, and chronic renal failure. New England Journal of Medicine 345, 1801-808.

Gomez, G., de Lusignan, S. and Gallagher, H. 2006: Chronic kidney disease: a new priority for primary care. The British Journal of General Practice 56, 908.

Gooch, K., Culleton, B.F., Manns, B.J., Zhang, J., Alfonso, H., Tonelli, M., Frank, C., Klarenbach, S., Hemmelgarn, B.R., Gooch, K., Culleton, B.F., Manns, B.J., Zhang, J., Alfonso, H., Tonelli, M., Frank, C., Klarenbach, S. and Hemmelgarn, B.R. 2007: NSAID use and progression of chronic kidney disease. American Journal of Medicine 120, e1-e7.

House, A.A., Silva Oliveira, S. and Ronco, C. 2007: Antiinflammatory drugs and the kidney. International Journal of Artificial Organs 30, 1042-46.

The Joint Specialty Committee on Renal Medicine of the Royal College of Physicians and the Renal Association. 2006: Chronic kidney disease in adults: UK guidelines for identification, management and referral. Retrieved March 2006 from www.renal.org/CKDguide/full/CKDprintedfull guide.pdf

NICE. 2008: CG73 Chronic kidney disease: Early identification and management of chronic kidney disease in adults in primary and secondary care. Retrieved September 2008 from www.nice.org.uk/nicemedia/pdf/ CG073FullGuideline.pdf

Stevens, P.E., O'Donoghue, D.J., de Lusignan, S., Van Vlymen, J., Klebe, B., Middleton, R., Hague, N., New, J. and Farmer, C.K.T. 2007: Chronic kidney disease management in the United Kingdom: NEOERICA project results. Kidney International 72, 92-99.

Wong, N.A. and Jones, H.W. 1998: An analysis of discharge drug prescribing amongst elderly patients with renal impairment. Postgraduate Medical Journal 74, 420-22. 